cell in the body to have a proper adequate and pure supply of blood, and this applies especially to cerebral neurones, as they are extremely sensitive to a change in environment. The author believes that he has obtained good results in the treatment of epilepsy by maintaining the alkalinity of the blood within physiological limits, but unfortunately he says it is impossible to elevate and maintain the alkalinity by the administration of drugs.

With reference to the leucocytes, there is a distinct leucocytosis, particularly after the attack. The author's general conclusions are as follows: (I) The alkalinity of the blood in the interparoxysmal period is lower than the average of the control cases; (2) the diminution is gradual and progressive and is more marked in those cases suffering from gastric catarrh and constipation; (3) there is a marked sudden and pronounced fall immediately prior to the onset of the fit; (4) there is a further fall in the alkalinity after the fit is over; this diminution is seen from three to ten minutes after the attack; (5) this after-diminution depends upon the duration and severity of the muscular twitching, and upon the degree of the alkalinity in the interparoxysmal period; (6) there is a gradual return of the blood to its normal alkalinity, which takes place in five to six hours, the rise being more marked in the first hour.

JELLIFFE.

\title{
LE NEVRAXE
}

(Vol. IV. Fasc. 3. 1903.)

8. On the Form and Development of the Protoplasmic Prolongations of the Spinal Cells in the Higher Vertebrates. T. Gerer.

9. Researches on the Central Acoustic Tracts: The Bulbo Mesencephalic Acoustic Tracts. VAN GEHUChTEN.

ro. The Inhibitory Fibers of the Heart belong to the Pneumogastric Nerve and not the Spinal Nerve. Van GeHuchten.

8. Form and Development of Protoplasmic Prolongations.-Dr. Geier has been studying the dendrites of the spinal cells of the horn fibers, using kittens and rabbits for investigations. Golgi's method was used. He finds that the dendrites of the anterior horns are smooth in contour and differ from the posterior horns which have an irregular contour. The spinal dendrites extend in a straight line, while those in the posterior horn are irregular and sinuous. The dendrites in the cells of the anterior horns have few and insignificant collateral appendices while those of the posterior horns are quite complex. There are many variations in the dendrites of the new-born animal as contrasted with those of the adult, for full details of which the reader is referred to the original.

9. The Upper Auditory Tract.-Van Gehuchten here presents results of an extended series of experiments performed on rabbits and studied by the Marchi method. Five series of experiments are given which lead Van Gehuchten to different conclusions than those heretofore held. In the first group of cases he tore the seventh nerve out through the stylomastoid rim. In this way the ventral fibers of the trapezoid body were involved. Serial sections showed that degenerated fibers were found passing into the trapezoid body vertically above and below the actual level of the lesions. In the trapezoid these fibers could be traced to the opposite side; they turn upwards, those ventrad passing in front of and those dorsad behind the trapezoid nucleus. These two degenerated bundles so united to form a compact strand of fibers which lay in contact with the lateral aspect of the inferior nucleus of the lateral lemniscus. Tracing this strand of fibers upward it was found to turn outward and then backwards finally entering and forming the more superficial part of the lat- 
eral fillet. From this place onward the fibers could be traced to the posterior quadrigemina where they terminated close to the superior nucleus of the lateral lemniscus. In the second series of cases, similar degenerations were found. In a third series a lesion was made by cutting downward and outward through the floor of the fourth ventricle, dividing both the dorsad and ventrad fibers of the corpus trapezoid. Similar conclusions as those already outlined were reached by this method of investigation. The fourth series of experiments was made to ascertain the cells of origin of the two strands of the corpus trapezoid tract. If a lesion was restricted entirely to the accessory nucleus of the eighth nerve degenerations corresponding to those just described resulted. On the question of the relation of the medullary stria in the secondary auditory path the author found that attempts to cut these on the floor of the fourth ventricle were unsuccessful but they were found destroyed in an experimented animal in which the seventh nerve had been torn out and had ruptured the acoustic strix. Degenerated fibers can be followed from the point of injury to their raphe where they crossed dorsad to the upper fibers and the trapezoid hody until they became posterior to the olivary nuclei. They then turned upwards, lying behind the dorsal trapezoid tract and internal and ventrad to the motor fifth nucleus. They entered the lateral lemmiscus at the upper border of the upper cerebral peduncle, being mingled with the fibers of the dorsal strand of the corpus trapezoid. They finally ended in the nucleus of the posterior quadrigemina itself, thus tracing the fibers of the secondary auditory path higher than those previously described. The author was unable to verify the opinion of Held that neither the dorsal nor ventrical auditory paths could be traced to the inferior corpora quadrigemina or cortex. Thus the anthor holds that the central auditory path is in reality a bulbo mesencephalic rather than a bulbo-cortical one. The illustrations are numerous, there being over 62 illustrations accompanying this extremely interesting and suggestive article.

10. Inhibitory Fibers of the Heart.-Van Gehuchten controverts the old idea that the inhibitory fibers of the heart arise in the spinal accessory and pass to join the vagus by its internal branch.

He concludes that (I) the inhibitory fibers of the heart do not come from the spinal accesory nerve but are derived from the vagus itself. (2) His investigations on the innervation of the larynx (see abstract of last number) go to prove that all of the bulbar fibers of the spinal accessory are distributed to the muscles of the larnyx; the eleventh nerve has an exclusive medullary origin and (4) the vagus gives off a superior and inferior system of fibers exclusively motor, including motor fibers destined to all the muscles of the larynx exclusive of the crico-thyroid, which is supplied by the inferior laryngeal nerve and a secondary series of inhibitory fibers to the heart.

JELLIFFE.

REVUE DE PSYCHIATRIE ET DE PSYCHOLOGIE EXPERIMENTALE

(January, I903.)

I. Tests in Psychopathology. En. Toulouse and N. Pí́ron.

2. Experimental Researches on the Psychology of Memories. N. VASChIDE and CL. VurPas.

3. Study of Morbid Obsessions.

I. Tests in Psychopathology.-The aim of this article is to indicate the lines along which the development of psychopathological tests must proceed if they are to be more exact and comparable. In their introduction the authors critically review the work of Guicciardi and Ferrari and of Tamburini, Badaloni and Brugia. They then proceed to a brief statement 